\title{
Magnetic resonance arthrography: what is its importance in the present day?
}

\author{
Artrorressonância magnética: qual a sua importância nos dias atuais?
}

\section{Guinel Hernandez Filho ${ }^{1}$}

There have been recent advances in the evolution of magnetic resonance imaging (MRI), including the use of 3.0 T machines in clinical practice, as well as improvements in the sequences and protocols employed in the examination of the musculoskeletal system ${ }^{(\mathbf{1})}$. Those advances have given rise to a question: what is the real need for minimally invasive diagnostic procedures, specifically magnetic resonance arthrography (MRA), in the assessment of intra-articular disorders?

The MRA examination has proven to be a diagnostic method of great sensitivity and specificity for the evaluation of conditions that affect intra-articular structures, such as instability of the capsuloligamentous structures of the shoulder, providing a more detailed evaluation of the changes to the articular cartilage surface and glenoid labrum ${ }^{(2,3)}$. In relation to the hip joint, MRA has also been the object of studies of femoroacetabular impingement, in which it has been shown to have excellent sensitivity and specificity for the staging of lesions of the articular cartilage and labrum ${ }^{(4)}$. Even in situations in which conventional MRI can meet the diagnostic needs in a broad sense, such as studies of the knee, MRA can still play a role in specific aspects, such as the postoperative evaluation of meniscal lesions ${ }^{(5)}$. Therefore, it can be said that MRA is still indicated for the diagnosis of some specific intra-articular conditions, including those affecting other joints, such as the elbow, wrist, and ankle.

The MRA procedure requires specific management of the patient, with a larger team of professionals; in most cases, MRA is used in conjunction with another imaging modality ( $X$ ray, ultrasound, or computed tomography) to guide the puncture, as well as requiring the investment of additional time on the part of the radiologist ${ }^{(6,7)}$. Albeit a minimally invasive procedure, MRA is not totally free of complications, which can include infection, reaction to the contrast medium, and post-puncture pain ${ }^{(\mathbf{8})}$. In addition, patients can become anxious when they are informed of the details of the procedure ${ }^{(9)}$, which can therefore be suspended due to non-consent.

Due to the greater complexity of the MRA, there have been various studies comparing conventional MRI and MRA, in terms of their diagnostic efficacy. Although some authors found no significant difference between the two methods ${ }^{(\mathbf{1 0})}$, the vast majority of such studies demonstrated the superiority

1. MD, Radiologist for the Grupo Musculoesquelético da Teleimagem/HCor, Grupo Alta/DASA, Attending Physician at Santa Casa de São Paulo, São Paulo, SP, Brazil. E-mail: guinel31@gmail.com. of MRA for the evaluation of lesions of the articular cartilages and labra, especially of those in the shoulder and hip ${ }^{\mathbf{( 1 1 , 1 2 )}}$.

Some studies have also helped demystify the topic of patient discomfort during MRA. In an article published in this issue of Radiologia Brasileira, Nascimento et al. ${ }^{\text {(13) }}$ concluded, on the basis of the responses obtained on questionnaires applied before and after the MRA examination, that the procedure is less painful than patients expect. The authors also described a novel technique in which the puncture is guided by conventional radiography.

It should be borne in mind that the techniques involved in the use of new MRI equipment and sequences, together with the continuing education and specialization of radiologists, have helped reduce the number of MRA exams. However, there are still evidence-based formal indications for the use of this imaging modality, which can be performed with relative safety and comfort for the patient.

\section{REFERENCES}

1. Chagas-Neto FA, Nogueira-Barbosa MH, Lorenzato MM, et al. Diagnostic performance of 3D TSE MRI versus 2D TSE MRI of the knee at $1.5 \mathrm{~T}$, with prompt arthroscopic correlation, in the detection of meniscal and cruciate ligament tears. Radiol Bras. 2016;49:69-74.

2. Honda E, Aihara AY, Natour J, et al. Artro-ressonância do ombro na instabilidade anterior. Rev Bras Reumatol. 2006;46:214-8.

3. Simão MN, Vinson EN, Spritzer CE. Magnetic resonance imaging evaluation of meniscoid superior labrum: normal variant or superior labral tear. Radiol Bras. 2016;49:220-4.

4. Sutter R, Zubler V, Hoffmann A, et al. Hip MRI: how useful is intraarticular contrast material for evaluating surgically proven lesions of the labrum and articular cartilage? AJR Am J Roentgenol. 2014;202:160-9.

5. Ciliz D, Ciliz A, Elverici E, et al. Evaluation of postoperative menisci with MR arthrography and routine conventional MRI. Clin Imaging. 2008;32:212-9.

6. Chung CB, Dwek JR, Feng S, et al. MR arthrography of the glenohumeral joint: a tailored approach. AJR Am J Roentgenol. 2001;177:217-9.

7. Berná-Serna JD, Redondo MV, Martinez F, et al. A simple technique for shoulder arthrography. Acta Radiol. 2006;47:725-9.

8. Saupe N, Zanetti M, Pfirrmann CWA, et al. Pain and other side effects after MR arthrography: prospective evaluation in 1085 patients. Radiology. 2009; 250:830-8.

9. Binkert CA, Zanetti M, Hodler J. Patient's assessment of discomfort during MR arthrography of the shoulder. Radiology. 2001;221:775-8.

10. Mintz DN, Hooper TR, Connell DA, et al. Magnetic resonance imaging of the hip: detection of labral and chondral abnormalities using non-contrast imaging. Arthroscopy. 2005;21:385-93.

11. Magee T. 3-T MRI of the shoulder: is MR arthrography necessary? AJR Am J Roentgenol. 2009;192:86-92.

12. Sutter R, Zubler V, Hoffmann A, et al. Hip MRI: how useful is intraarticular contrast material for evaluating surgically proven lesions of the labrum and articular cartilage? AJR Am J Roentgenol. 2014;202:160-9.

13. Nascimento PCX, Amaral AM, Almeida JRM. Magnetic resonance arthrography of the shoulder: a painful procedure? Radiol Bras. 2018;51:81-6.

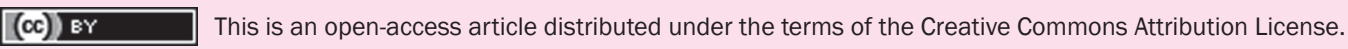

